\title{
EFEITO AGUDO DO INDACATEROL EM DESFECHOS CLÍNICOS E FUNCIONAIS NOS PORTADORES DE DOENÇA PULMONAR OBSTRUTIVA CRÔNICA (DPOC): UM ENSAIO CLÍNICO RANDOMIZADO
}

\author{
Thiago Fróes Batista Ribeiro Acadêmico do Curso de Medicina, Escola Bahiana \\ de Medicina e Saúde Pública. \\ Fernanda Warken Rosa Docente Adjunto DE, Curso de Fisioterapia, \\ Universidade do Estado da Bahia. \\ Tiago Albertassi Blunck Resende Acadêmico do Curso de Medicina, Escola Bahiana \\ de Medicina e Saúde Pública. \\ Aquiles Assunção Camelier Docente Adjunto, Escola Bahiana de Medicina e \\ Saúde Pública. Centro de Estudos Egas Moniz, \\ Hospital Português-Ba.
}

\begin{abstract}
Resumo
Introdução:Os broncodilatadores são fundamentais para o tratamento da doença pulmonar obstrutiva crônica (DPOC). O indacaterol é broncodilatador beta-2-agonista de longa ação recentemente aprovado para utilização na DPOC, que tem como principal característica referida pelos fabricantes um rápido início de ação. Objetivo: Avaliar o efeito agudo do indacaterol em portadores de DPOC. Métodos: Foi realizado um ensaio clínico randomizado, duplo cego, controlado com placebo utilizando indacaterol $150 \mathrm{mcg}$ inalado em portadores de DPOC. Os desfechos avaliados foram o VEF1 e a CVF (obtidos da espirometria), frequência cardíaca e respiratória, pressão arterial, $\mathrm{SpO}_{2}$, sensação de dispneia e o teste do degrau. Resultados: Quarenta pacientes foram selecionados. Observou-se que não foi notada broncodilatação significante entre os grupos indacaterol e Placebo aos 5, 15 e 30 minutos seja em relação ao $V F_{1}$ ou a CVF. Diferenças estatísticas também não foram notadas para as variáveis secundárias como $\mathrm{SpO}_{2}(\mathrm{p}=0,89), \mathrm{FR}(\mathrm{p}=0,88), \mathrm{FC}(\mathrm{p}=0,31)$, pressão arterial $(\mathrm{p}=0,85)$, dispneia $(\mathrm{p}=0,60)$ e número de degraus subido ao final de 6 minutos $(0,72)$. Conclusão: Em portadores de DPOC estáveis o indacaterol não produziu modificações nos desfechos avaliados em até 30 minutos após a sua utilização.
\end{abstract}

Palavras-chaves: DPOC; Espirometria; Placebo; Broncodilatadores; Ensaio Clínico Randomizado; Tolerância ao Exercício.

\section{ACUTE EFFECT OF INDACATEROL IN CLINICAL AND FUNCTIONAL OUTCOMES IN PATIENTS WITH CHRONIC OBSTRUCTIVE PULMONARY DISEASE (COPD): A RANDOMIZED CONTROLLED TRIAL}

\begin{abstract}
Introduction:Bronchodilatorsarethemaintreatmentofchronicobstructivepulmonarydisease(COPD).

Theindacaterol is a long-acting beta-2-agonist bronchodilatorrecentlyapproved for usein COPD, whose maincharacteristicreferred toby manufacturersa rapid onset ofaction.Objective: To evaluatethe acute effect ofindacaterolin COPD patients. Methods: A randomized,double-blind, placebo-controlled study was conducted, using150 mcginhaledindacaterolin patients withCOPD. The outcomes assessed wereFEV1 and FVC(obtained through spirometry), heart and respiratory rate,blood pressure, SpO2, dyspnea sensationandstep test. Results:Fortypatients were enrolled.It was observed thatnosignificantbronchodilationwas notedbetween the indacateroland Placebogroups at 5,15 and 30 minutesiscompared to theFEV1 orFVC.Statistical differenceswere notnoted forthesecondary variablesasSpO2( $\mathrm{p}=0.89), \operatorname{FR}(\mathrm{p}=0.88), \mathrm{HR}(\mathrm{p}=0.31)$, blood pressure $(\mathrm{p}=0.85)$, dyspnea(p =0.60) and number of stepsclimbedafter 6minutes (0.72). Conclusion:In patients withstableCOPDindacaterolproduced nochangesin the outcomes assessedwithin 30 minutesafter use.
\end{abstract}

Keywords: COPD; Spirometry; Placebo; Bronchodilators; Randomized Clinical Trial; Exercise Tolerance. 
Ribeiro et al. Efeito agudo do indacaterol em desfechos clínicos e funcionais nos portadores de doença pulmonar obstrutiva crônica (DPOC): um ensaio clínico randomizado.

\section{INTRODUÇÃO}

O tratamento para pacientes portadores de Doença Pulmonar Obstrutiva Crônica (DPOC) visa aliviar os sintomas, prevenir a progressão da doença, melhorar a tolerância ao exercício, melhorar o estado de saúde, prevenir complicações e exacerbações e principalmente reduzir a mortalidade. ${ }^{(1)}$

Para avaliar a eficácia destes tratamentos, se utilizam desfechos importantes para estes pacientes: a intensidade de broncodilatação, medidos através do Volume Expiratório Forçado no $1^{\text {o }}$ segundo (VEF1) e da Capacidade Vital Forçada (CVF), a Saturação do Pulso de Oxigênio ( $\mathrm{SpO} 2)$, a sensação de dispneia, capacidade de exercício e parâmetros cardiovasculares durante o esforço, bem como a qualidade de vida. ${ }^{(1)}$

O indacaterol é o medicamento broncodilatador mais recentemente indicado para a DPOC estável. ${ }^{(2,3)} \mathrm{O}$ mesmo é considerado um beta-2-agonista de longa duração, mas que possui início de ação rápida, o que o diferencia em relação a outros broncodilatadores de longa ação disponíveis. ${ }^{(5,6,7)}$

O objetivo do presente estudo foi avaliar o efeito agudo do indacaterol em portadores de DPOC, utilizando desfechos clínicos e funcionais como a espirometria, a sensação de dispneia e o teste do degrau, além da frequência cardíaca e respiratória, pressão arterial e $\mathrm{SpO}_{2}$.

\section{METODOLOGIA}

A coleta de dados foi realizada no Complexo Hospitalar Universitário Professor Edgard Santos/Ambulatório Magalhães Neto (CHUPES), Salvador-Bahia, no período entre março a abril de 2012. Como critérios de inclusão foram utilizados o Diagnóstico de DPOC (conforme normativas GOLD), idade maior ou igual a 40 anos. Foram excluídos indivíduos com presença de exacerbação da DPOC (em um tempo igual ou inferior a 30 dias), presença de alergia ou contra indicação ao indacaterol.

Foi delineado um estudo randomizado, controlado, duplo cego, controlado com placebo. Os pacientes portadores de DPOC foram randomizados para dois grupos de intervenção: um braço utilizou indacaterol 150 mcg inalado em preparação de pó seco através de um dispositivo de inalação específico (Onbrize-Novartis Farmacêutica®) e o outro braço

Revista Pesquisa em Fisioterapia, Salvador, dez. 2012; 2(2): 83-91. 
Ribeiro et al. Efeito agudo do indacaterol em desfechos clínicos e funcionais nos portadores de doença pulmonar obstrutiva crônica (DPOC): um ensaio clínico randomizado.

utilizou o placebo. A sequência aleatória foi obtida de um programa de randomização gratuito da internet (www.randon.org) e os pacientes foram selecionados a partir da abertura de envelopes selados previamente ordenados.

A espirometria foi realizada em um aparelho portátil modelo EasyOne® (Medical Technologies, Chelmsford, Massachusetts and Zurich, Switzerland). Os procedimentos foram realizados de acordo com as diretrizes brasileiras para o estudo da função pulmonar, versão 2002. ${ }^{(8)}$ A espirometria foi realizada quatro vezes, nos tempos 0 minuto, e as outras três vezes após acontecer à intervenção com uso de indacaterol 150mcg ou placebo (aos 5, 15 e 30 minutos).

A oximetria do pulso do oxigênio $\left(\mathrm{SpO}_{2}\right)$ foi avaliada com o oxímetro modelo 300 da Healthdyne. O teste do degrau foi padronizado em 6 minutos, no qual o paciente determinava a cadência de subida e descida dos degraus. O mesmo foi realizado em dois momentos, antes da intervenção, ou após o trigésimo minuto desta.Os valores da frequiência cardíaca (bpm), frequiência respiratória (ipm) e SpO2 (\%), além de sensação de dispneia (através da escala de BORG), foram colhidos no repouso e em pé antes e logo após a realização do teste do degrau.A dipsneia foi avaliada utilizando-se a escala de dispneia do Medical Research Council (MRC). ${ }^{(9)}$

Para a realização do cálculo amostral, foi utilizada a detecção da diferença de $200 \mathrm{ml}$ de $\mathrm{VEF}_{1}$ entre os grupos, considerada clinicamente importante, assumindo um erro do Tipo 1 ( ou valor de p) menor que 0,05 e um erro do tipo 2 ou $\beta$ de $20 \%$. Foram então estimados a avaliação de 17 sujeitos em cada braço de intervenção. Estimando a possibilidade de perdas durante o estudo, foram estudados mais três pacientes em cada braço (aproximadamente $20 \%$ da amostra calculada para cada braço do estudo). No total foram então previstos a inclusão de 40 pacientes.

Os valores numéricos foram expressos em média e desvio padrão. Variáveis categóricas foram expressas como percentuais. As diferenças entre os valores de $\mathrm{VEF}_{1}, \mathrm{CVF}$, frequência cardíaca, frequência respiratória, saturação de oxigênio e sensação de dispnéia com escala de BORG foram avaliadas através do Teste de Mann Whitney, após a análise da distribuição dos dados com o teste de Kolmogorov-Smirnov. Para cálculo da diferença entre os valores percentuais, foi utilizado o teste de Qui-quadrado. Para a análise estatística foi utilizado o programa SPSS versão 14 . Um valor de $\mathrm{p}<0,05$ foi considerado significante.

Revista Pesquisa em Fisioterapia, Salvador, dez. 2012; 2(2): 83-91 
Ribeiro et al. Efeito agudo do indacaterol em desfechos clínicos e funcionais nos portadores de doença pulmonar obstrutiva crônica (DPOC): um ensaio clínico randomizado.

Foi obtida a aprovação do Comitê de Ética em Pesquisa da Fundação Bahiana para o Desenvolvimento das Ciências; Escola Bahiana de Medicina e Saúde Pública (protocolo 214/2011 e ofício 34/2012).O presente estudo foi registrado no site de ensaios clínicos da Base Nacional de Ensaios Clínicos da ANVISA - Brasil (www.ensaiosclinicos.gov.br) n ${ }^{\circ} 617$.

\section{RESULTADOS}

Foram selecionados um total de 40 pacientes que preencheram os critérios de inclusão e exclusão. Destes pacientes, 20 foram sorteados para realizar o uso do indacaterol $150 \mathrm{mcg}$ e os outros 20 para a utilização do placebo (grupo placebo). Os dados clínicos e demográficos do grupo que usou indacaterol e placebo estão listados na Tabela 1.

Tabela 1- Dados clínicos e demográficos do grupo indacaterol e placebo, da amostra dos pacientes portadores de DPOC.

\begin{tabular}{llll}
\hline & Indacaterol & Placebo & $p$ \\
\hline Idade (anos) & $68,2 \pm 8,7$ & $63,9 \pm 6,1$ & 0,12 \\
Masculino/Feminino & $13(65 \%) / 7(35 \%)$ & $15(67 \%) / 5(33 \%)$ & 0,49 \\
$\mathrm{CVF}^{\phi}(\mathrm{L})$ & $2,1 \pm 0,8$ & $2,5 \pm 0,5$ & 0,06 \\
$\mathrm{CVF}(\%)$ & $68,6 \pm 19,6$ & $74,9 \pm 15,9$ & 0,53 \\
$\mathrm{VEF}_{1}{ }^{*}(\mathrm{~L})$ & $1,3 \pm 0,5$ & $1,3 \pm 0,5$ & 0,75 \\
$\mathrm{VEF}_{1}(\%)$ & $49,8 \pm 14,1$ & $46,9 \pm 18,1$ & 0,51 \\
$\mathrm{VEF}_{1} / \mathrm{CVF}$ & $0,4 \pm 0,1$ & $0,4 \pm 0,1$ & 0,66 \\
$\mathrm{SpO}_{2}{ }^{-}$ & $92,0 \pm 5,7$ & $92,0 \pm 5,8$ & 0,92 \\
$\mathrm{Dispnéia} \mathrm{MRC}^{2}$ & $2,3 \pm 1,2$ & $2,1 \pm 1,2$ & 0,60 \\
\hline
\end{tabular}

${ }^{\phi} \mathrm{CVF}$ : Capacidade Vital Forçada, pós broncodilatador. ${ }^{*} \mathrm{VEF}_{1}$ : Volume expiratório forçado no primeiro segundo, pós broncodilatador. $\mathrm{SpO}_{2:}$ Saturação de Oxigênio. ${ }^{\alpha} \mathrm{MRC}$ : Medical Research Council.

Analisando o grupo dos 40 pacientes como um todo, foi visto que a média de idade dos pacientes foi de $66,05 \pm$ 7,72 anos, no qual participaram 28 pacientes do sexo masculinos contra 12 pacientes do sexo feminino. Esses pacientes foram submetidos à realização da espirometria no qual demonstrou uma média de 2,32 $\pm 0,68$ litros para a medida do CVF(L) e uma média de 1,27 $\pm 0,51$ litros para a medida do $\operatorname{VEF}_{1}(\mathrm{~L})$. Obtiveram-se também valores de $71,58 \pm 18,00$ por cento e 48,44 $\pm 15,94$ por cento para seus respectivos valores em percentual. Observou-se um valor de Tiffeneau com uma média de 0,43 $\pm 0,13$ litros entre os pacientes dessa pesquisa e valores de $\mathrm{SpO}_{2}$ numa média de 92,00 \pm 5,623 por cento de saturação. De acordo com o MRC a sensação de dispneia alcançou uma média de $2,17 \pm 1,19$ pontos. O tempo total de tabagismo foi ,em média, 36,70 \pm 15,98 anos. 
Ribeiro et al. Efeito agudo do indacaterol em desfechos clínicos e funcionais nos portadores de doença pulmonar obstrutiva crônica (DPOC): um ensaio clínico randomizado.

Para avaliar o efeito do indacaterol da amostra estudada, foram avaliados os incrementos (em ml) do VEF1 e a CVF ao início da pesquisa, aos 5, 15 e 30 minutos. Em relação ao VEF1, aos 5 minutos, houve um incremento de $0,006 \pm 0,19 \mathrm{ml}$ no grupo que usou indacaterol, em relação ao grupo placebo (incremento de 0,23 $\pm 0,16 \mathrm{ml}, \mathrm{p}=0,45$ ). Aos 15 minutos, houve um incremento de $-0,4 \pm 0,18 \mathrm{ml}$ no grupo que usou indacaterol, em relação ao grupo placebo (incremento de $0,04 \pm 0,249 \mathrm{ml}, \mathrm{p}=0,41$ ). $\mathrm{E}$, finalmente, aos 30 minutos, houve um incremento de $-0,0075 \pm 0,17 \mathrm{ml}$ no grupo que usou indacaterol, em relação ao grupo placebo (incremento de 0,06 $\pm 0,32 \mathrm{ml}, \mathrm{p}=0,9$ ).

Em relação a CVF, aos 5 minutos, houve um incremento de $-0,35 \pm 0,20 \mathrm{ml}$ no grupo que usou indacaterol, em relação ao grupo placebo (incremento de $-0,05 \pm 0,11 \mathrm{ml}, \mathrm{p}=0,83$ ). Aos 15 minutos, houve um incremento de $-0,08 \pm 0,2 \mathrm{ml}$ no grupo que usou indacaterol, em relação ao grupo placebo (incremento de $-0,03 \pm 0,15 \mathrm{ml}, \mathrm{p}=0,29$ ). Aos 30 minutos, houve um incremento de $-0,04 \pm 0,21 \mathrm{ml}$ no grupo que usou indacaterol, em relação ao grupo placebo (incremento de $-0,03 \pm 0,13 \mathrm{ml}, \mathrm{p}=0,37$ ).

Além de parâmetros de espirometria, também foram estudados o efeito agudo do indacaterol em relação à dispneia e sensação de cansaço nas pernas (medidos através da escala de BORG) no teste do degrau antes e após a intervenção medicamentosa (indacaterol $150 \mathrm{mcg}$ ) ou uso do placebo, bem como a quantidade de degraus subida, medidas de tensão arterial, FC, FR e a SpO2 (\%) antes e após a intervenção (Tabelas 2 e 3). Nestes testes foram avaliados a variação da sensação de dispneia antes e ao final do teste do degrau, antes e após a intervenção com uso do broncodilatador indacaterol ou placebo. Em seguida, foi analisado se houve variação da sensação de dispneia e da sensação de cansaço nas pernas antes e após a intervenção.

Tabela 2 -Dados de SpO2, FR, FC, TAS e TAD antes e após a realização do teste do degrau antes das intervenções indacaterol e placebo.

\begin{tabular}{|c|c|c|c|}
\hline & caterol & Controle & $P$ \\
\hline $\mathrm{SpO}_{2}{ }^{\mathrm{a}}$ basal & $95,4 \pm 2,2$ & $98,0 \pm 24,7$ & 0,97 \\
\hline $\mathrm{SpO}_{2}$ final & $92,0 \pm 5,0$ & $94,4 \pm 4,0$ & 0,27 \\
\hline $\mathrm{FR}^{\mu}$ basal & $19,3 \pm 3,4$ & $19,9 \pm 5,0$ & 0,54 \\
\hline FR final & $24,3+5,8$ & $23,2+5,1$ & 0,44 \\
\hline $\mathrm{FC}^{\mathrm{e}}$ basal & $77,5 \pm 14,7$ & $72,5 \pm 11,5$ & 0,38 \\
\hline FC final & $91,6+21,4$ & $94,6+18,3$ & 0,52 \\
\hline $\mathrm{TAS}^{¥}$ basal & $129,0 \pm 17,1$ & $133,1 \pm 18,6$ & 0,69 \\
\hline TAS final & $140,3 \pm 23,5$ & $138,6 \pm 28,5$ & 0,68 \\
\hline $\mathrm{TAD}^{\square}$ basal & $79,5 \pm 12,3$ & $81,0 \pm 8,5$ & 0,68 \\
\hline TAD Final & $78,5+10,9$ & $80,5+12,1$ & 0,62 \\
\hline
\end{tabular}


Ribeiro et al. Efeito agudo do indacaterol em desfechos clínicos e funcionais nos portadores de doença pulmonar obstrutiva crônica (DPOC): um ensaio clínico randomizado.

${ }^{a} \mathrm{SpO}_{2}=$ Saturação de Oxigênio ${ }^{\mu} \mathrm{FR}=$ Frequência Respiratória ${ }^{\hat{e}} \mathrm{FC}=$ Frequência Cardíaca ${ }^{\ddagger} \mathrm{TAS}=$ Tensão Arterial Sistólica ${ }^{\square} \mathrm{TAD}=$ Tensão Arterial Diastólica

Tabela 3 -Dados de SpO2, FR, FC, TAS e TAD antes e após a realização do teste do degrau, 30 minutos após as intervenções indacaterol e placebo.

\begin{tabular}{llll}
\hline & \multicolumn{1}{c}{ Indacaterol } & Controle & $p$ \\
\hline SpO2 ${ }^{\text {a }}$ basal & $95,4 \pm 1,50$ & $95,1 \pm 3,3$ & 0,79 \\
SpO2 final & $92,0 \pm 5,7$ & $92,0 \pm 5,8$ & 0,89 \\
FR $^{\mu}$ basal & $18,0 \pm 3,4$ & $19,5 \pm 4,3$ & 0,24 \\
FR final $_{F C}^{\hat{\imath}}$ basal & $24,2 \pm 4,6$ & $24,5 \pm 5,1$ & 0,88 \\
FC final $_{\text {TAS }}^{¥}$ basal & $78,8 \pm 14,3$ & $75,0 \pm 15,7$ & 0,51 \\
TAS final & $88,2 \pm 20,0$ & $93,3 \pm 17,3$ & 0,31 \\
TAD & $120,0 \pm 30,3$ & $125,5 \pm 16,7$ & 0,51 \\
TAD Final & $140,4 \pm 22,4$ & $137,3 \pm 19,3$ & 0,85 \\
& $77,9 \pm 11,1$ & $76,5 \pm 7,5$ & 0,93 \\
\hline
\end{tabular}

${ }^{\alpha} \mathrm{SpO}_{2}=$ Saturação de Oxigênio $\quad{ }^{\mu} \mathrm{FR}=$ Frequência Respiratória ${ }^{k} \mathrm{FC}=$ Frequência Cardíaca ${ }^{\ddagger} \mathrm{TAS}=$ Tensão Arterial Sistólica ${ }^{\square} \mathrm{TAD}=$ Tensão Arterial Diastólica

No teste do degrau também foi observado o número de degraus que cada paciente conseguia subir antes da intervenção e após a intervenção para que logo em seguida pudéssemos calcular a diferença entre os degraus subidos nesses dois momentos, avaliando desta forma o efeito broncodilatador agudo que o indacaterol poderia produzir nos participantes (Tabela 4).

Tabela 4 - Números totais de degraus subidos durante o teste do degrau pelos grupos indacaterol e placebo, antes e após a intervenção.

\begin{tabular}{|c|c|c|c|}
\hline Número de degraus subidos & Indacaterol & Placebo & $P$ \\
\hline Degraus subidos antes da intervenção. & $65,5 \pm 30,4$ & $79,0 \pm 29,4$ & 0,15 \\
\hline $\begin{array}{l}\text { Degraus subidos depois } \quad \mathrm{da} \\
\text { intervenção. }\end{array}$ & $75,2 \pm 29,0$ & $90,7 \pm 29,6$ & 0,11 \\
\hline $\begin{array}{l}\text { Diferença de degraus subidos entre } \\
\text { antes e depois da intervenção. }\end{array}$ & $9,7 \pm 9,1$ & $11,7 \pm 14,8$ & 0,72 \\
\hline
\end{tabular}

\section{DISCUSSÃO}

A DPOC é uma doença que pode acometer muito a qualidade de vida das pessoas, principalmente decorrente da presença de sintomas como a dispneia e a intolerância ao exercício. ${ }^{(1,10)}$ Existem diversas intervenções na literatura que podem modificar estes sintomas, 
Ribeiro et al. Efeito agudo do indacaterol em desfechos clínicos e funcionais nos portadores de doença pulmonar obstrutiva crônica (DPOC): um ensaio clínico randomizado.

como a utilização de medicamentos broncodilatadores e a prescrição de exercícios sob a forma da reabilitação pulmonar: ${ }^{(1,10)}$ Dentre os broncodilatadores mais utilizados estão os de longa duração que proporciona um efeito mais duradouro ao paciente ao decorrer do dia, gerando assim um alivio mais acentuado da dispneia. ${ }^{(1,10)}$ Entretanto os tempos de início de ação (broncodilatação) capaz de gerar um alívio da dispneia destes medicamentos podem variar de 20 a 30 minutos. ${ }^{(1)} \mathrm{O}$ indacaterol, conforme dados da literatura (colocar referências), seria capaz de promover um início de broncodilatação mais precoce (nos primeiros $05 \mathrm{~min}$ ), o que poderia proporcionar um alívio imediato na sensação de dispneia e no aumento da tolerância ao exercício, que na DPOC são justificados pela redução de limitação ao fluxo aéreo (que pode ser mensurado pelo VEF1) e consequente redução de hiperinsuflação pulmonar (mensurados indiretamente pela CVF). ${ }^{(4)}$ Uma outra vantagem teórica da ação rápida de broncodilatação seria a percepção e associação do alívio da dispneia e da melhor tolerância ao exercício ao uso de medicamento, fator que poderia contribuir para adesão do tratamento a longo prazo. ${ }^{(10)}$

A análise demográfica e clínica demonstrou que a amostra estudada possuía semelhantes características das avaliadas em outros ensaios clínicos que avaliaram a DPOC, o que garante a generalização dos resultados. Os pacientes do presente estudo tinham, em sua maioria, uma doença em fase mais avançada (DPOC moderado a grave) e, portanto, tinham um elevado grau de comprometimento respiratório, levando a sensação de dispneia e a redução da capacidade de exercício, conforme demonstrados pelos testes do degrau e escala MRC.

Entretanto, a presença de broncodilatação aguda após o uso de indacaterol, não apresentou diferença estatisticamente significante do $\mathrm{VEF}_{1(\mathrm{~L})}$ entre o grupo teste e o grupo placebo. Estes achados sugerem que o indacaterol, apesar de gerar broncodilatação a longo prazo conforme evidenciado em estudos da literatura, no presente estudo não foi encontrada uma broncodilatação aguda (de até 30 minutos) suficiente para gerar uma melhora da função pulmonar. ${ }^{(2-7)}$ Uma outra hipótese a ser pensada é que a broncodilatação aguda inerente ao indacaterol poderia estar presente, mas por problemas de poder de amostra, talvez a quantidade de sujeitos observada não tenha sido suficiente para detectar tal diferença (incorrendo em um erro estatístico do tipo II).

Além de parâmetros de espirometria, neste estudo procurou-se avaliar variáveis mais próximas da compreensão e valorização por parte do paciente leigo. O teste do degrau é 
Ribeiro et al. Efeito agudo do indacaterol em desfechos clínicos e funcionais nos portadores de doença pulmonar obstrutiva crônica (DPOC): um ensaio clínico randomizado.

considerado um teste simples e capaz de promover a avaliação da capacidade funcional de portadores de DPOC, além de prover resultados adicionais de dispneia e adaptação cardiorrespiratória. ${ }^{(1)}$ Através do teste do degrau foi evidenciado que todos os pacientes experimentaram intolerância ao exercício, demonstrada através do aumento na FC, FR e dispneia após o término do esforço padronizado aos quais eles foram submetidos. Percebeuse, entretanto, através da análise estatística, que não houve diferença estatisticamente significante $(\mathrm{p}=0,15)$ entre o grupo indacaterol e grupo placebo quanto ao número de degraus subidos antes da intervenção, bem como de outros parâmetros avaliados (Fc, Fr, SpO2 e dispneia através da escala de BORG). Ficou evidenciado que o indacaterol não afetou de forma significante o desempenho ao exercício dos portadores de DPOC, quando comparados ao placebo.

Por outro lado, também não foram visualizados importantes efeitos colaterais de um medicamento beta-2-agonista, a taquicardia e elevação dos níveis tensionais, que podem decorrer da excessiva estimulação de beta receptores no músculo cardíaco ou esquelético. $\mathrm{O}$ que confere um ponto de segurança do ponto de vista cardiovascular, inclusive durante a realização de exercícios físicos.

O tamanho amostral pode ser considerado uma limitação deste estudo, devido a incapacidade de ter demonstrado um possivel, mas menos intenso, efeito na broncodilatação e na tolerância ao exercício em portadores de DPOC.

\section{CONCLUSÃO}

O indacaterol administrado em sujeitos portadores de DPOC não confere modificações agudas na broncodilatação, na sensação de dispneia ou na tolerância ao exercício quando comparados ao placebo.

\section{REFERÊNCIAS}

1. Rabe KF, Hurd S, Anzueto A, Barnes PJ, Buist SA, Calverley P, et al.Global strategy for the diagnosis, management, and prevention of chronic obstructive pulmonary disease: GOLD executive summary. American journal of respiratory and critical care medicine.

2007;176(6):532-55. Available at: http://www.ncbi.nlm.nih.gov/pubmed/17507545 [Accessed July 16, 2011]. 
Ribeiro et al. Efeito agudo do indacaterol em desfechos clínicos e funcionais nos portadores de doença pulmonar obstrutiva crônica (DPOC): um ensaio clínico randomizado.

2. Chowdhury BA, Seymour SM, Michele TM, Durmowicz AG, Liu D, Rosebraugh CJ. The Risks and Benefits of Indacaterol. The FDA's Review. New Eng J Med. 2011; 365: 24.

3. Beeh BE. Long-acting $\beta$-adrenoceptor agonists in the management of COPD: focus on indacaterol. Int J COPD. 2011; 6: 237-243.

4. Balint B, Watz H, Amos C, Owen R, Higgins M, Kramer B.Onset of action of indacaterol in patients with COPD: Comparison with salbutamol and salmeterol-fluticasone. Int J COPD. 2010;5:311-318.

5. Jones PW, Barnes N, Vogelmeier C, Lawrence D, KramerB.Efficacy of indacaterol in the treatment of patients with COPD. Prim Care Respir J. 2011; 20(4): 380-388.

http://dx.doi.org/10.4104/pcrj.2011.00066

6. Sturton RG, Trifilieff A, Nicholson AG, Barnes PJ.Pharmacological Characterization of Indacaterol , a Novel Once Daily Inhaled `` 2 Adrenoceptor Agonist , on Small Airways in Human and Rat Precision-Cut Lung Slices. Pharmacology. 2008;324 (1):270-275.

7. Ribeiro M, Chapman K. Comparative eficacy of indacaterol in chronic obstructive pulmonary disease. International Journal of COPD. 2012;7:145-152.

http://dx.doi.org/10.2147/COPD.S19805

8. Sociedade Brasileira de Pneumologia e Tisiologia. II Consenso Brasileiro sobre Doença pulmonar obstrutiva crônica. JBrasPneumol. 2002;28(Supl 3):1-238.

9. Nishimura K. Dyspnea Is a Better Predictor of 5-Year Survival Than Airway Obstruction in Patients With COPD. Chest. 2002.[Accessed March 9, 2012];121(5):1434-1440. Available at: http://www.chestjournal.org/cgi/doi/10.1378/chest.121.5.1434.

10. Qaseem A, Wilt TJ, Weinberger SE, Hanania NA, Criner G, Vander Molen T, et al. Diagnosis and Management of Stable Chronic Obstructive Pulmonary Disease: A Clinical Practice Guideline Update from the American College of Physicians, American College of Chest Physicians, American Thoracic Society, and European Respiratory Society. Ann of internal medicine. 2011;155(3):179-191. Available at: http://www.ncbi.nlm.nih.gov/pubmed/21810710.

11. Divo M, Pinto-Plata V.Role of exercise in testing and in therapy of COPD.Med Clin North Am. 2012 Jul;96(4):753-766. 